\title{
"DORT UNTEN" - Die frühneuhochdeutsche Handschrift in Doderers Roman Die Dämonen und ihre Übersetzung ins Tschechische
}

\section{"DOWN THERE" - The Early New High German Text in Doderers Novel Die Dämonen and its Translation into Czech Language}

Anna Kovaříková

\begin{abstract}
This article documents an attempt to translate one chapter of a novel Die Dämonen by Heimito von Doderers from Early New High German into Czech. In its first and main part, it describes how the author treated this historical language, the means he used and to what purpose, and also, the method that would be chosen for its translation into Czech. The second part of the article deals with the sensory perception in this particular passage and how the individual senses are communicated to the reader.
\end{abstract}

Keywords

Heimito von Doderer; Die Dämonen; Early High New German; translation 
Für wohl jeden, der Heimito von Doderers umfangreichen Roman Die Dämonen liest, dürfte das fünfzigseitige Kapitel „DORT UNTEN “1 von besonderem Interesse sein, zumindest in formaler Hinsicht. Es gibt vor, ein altes, in der Romanhandlung kurz zuvor entdecktes Manuskript aus dem 16. Jahrhundert zu sein, das im seinerzeit gängigen Frühneuhochdeutsch verfasst ist.

Dieser Beitrag befasst sich damit, wie Doderer das Frühneuhochdeutsche darin verwendet, welche sprachlichen Mittel er einsetzt und warum, wie ein derart archaisierter Text ins Tschechische zu übersetzen und welches tschechische Äquivalent zum Frühneuhochdeutschen zu wählen ist, um einerseits die Besonderheit des Originals angemessen wiederzugeben und andererseits einer tschechischen Leserschaft keine allzu großen Verständnisprobleme zu bereiten. Ich werde mich bei den Beispielen und Leseproben auf eine Textpassage beschränken, die mir sowohl sprachlich als auch thematisch reizvoll zu übersetzen erschien. Im dritten Teil des Beitrags werde ich die Art und Weise untersuchen, wie Doderer im fraglichen Abschnitt, der in diesem Sinne besonders interessant ist, mit Sinneseindrücken - Geruchs- und Gesichts-, insbesondere Farbwahrnehmungen - arbeitet.

In Doderers Roman heißt es, dass die Handschrift von einem ehemaligen „Edelknabe[n]“ im „Dienste“ des Burgherrn „Achaz von Neudegg“, der sich „Ruodlieb von der Vläntsch" nennt, im Jahr 1517 verfasst worden sei und Ereignisse aus dem Jahr 1463 wiedergebe. ${ }^{2}$ Damals habe Ruodliebs Herr zwei Frauen der Hexerei bezichtigt und in seiner Burg festsetzen lassen. Achaz habe die beiden, die Witwe eines Bürgermeisters, genannt Agnes Stoecherin, ${ }^{3}$ und deren etwas jüngere Verwandte Maria, die meist als „Gevattrin" bezeichnet wird, ${ }^{4}$ auf einer Reise in einer Herberge getroffen, wo er sich mit den Frauen unterhalten und reichlich Wein mit ihnen getrunken habe. Im Lauf des Abends habe die Bürgermeisterwitwe aus ihrer Kammer ein Gewürz geholt, das - in den Wein getan - gegen seine Müdigkeit und Trunkenheit geholfen habe. Achaz nimmt dies zum Vorwand, die beiden Frauen, die ihn erotisch ansprechen, seinen Avancen jedoch nicht entgegengekommen sind, festzunehmen und in seiner Burg gefangen zu setzen. Er initiiert - in keiner Weise zuständig oder befugt - einen Prozess und lässt sie von einem improvisierten Gericht der Hexerei anklagen. Im Zuge der ,Ermittlungen“ werden sie befragt, verschiedenen schmählichen Prozeduren unterworfen und zuletzt auch gefoltert. Ruodlieb ist daran, zunächst als Protokollführer, zwar unfreiwillig, aber aktiv beteiligt. Letztlich dienen die ,peinlichen Befragungen' aber nur der Befriedigung der sexuellen Bedürfnisse des Burgherrn, denn auf seine Anweisung hin dürfen die entkleideten Frauen durch die - inszenierte - Folter nicht verletzt werden. Ruodlieb und sein Freund Heimo, welche die Marter gemeinsam durchführen, treffen - auf Vorschlag der beiden Frauen - schließlich eine Abmachung: Die Knappen mögen ihrem Herrn erklären, dass sie an ihnen keine Hexenmale gefunden hätten, im Gegenzug würden die Frauen ihnen

1 Vgl. Doderer, Heimito von: Die Dämonen. Nach der Chronik des Sektionsrates Geyrenhoff. München 1995, S. 757-806.

2 Doderer, Die Dämonen (Anm. 1), S. 731.

3 Vgl. Doderer, Die Dämonen (Anm. 1), S. 765 u. 777.

4 Vgl. Doderer, Die Dämonen (Anm. 1), S. 773 (Name) bzw. 780, 783, 784, 785, 787, 790 u. 791 sowie 781 u. 792 („Gevatterin“). 
dafür in ihrer Kammer sexuell zu Diensten sein. Im weiteren Verlauf kommen die Frauen frei, werden entschädigt und in die Obhut der Obrigkeit übergeben.

Doderer verfasste den frühneuhochdeutschen Text mit bewundernswerter historischer Treue und unter Wahrung sprachlicher Spezifika auf allen Ebenen. Am auffälligsten zeigt sich das in der Schreibung und Lautgestalt, wo es große Unterschiede zur gegenwärtigen Sprachstufe gibt, verglichen etwa mit der Morphologie, wo die Unterschiede zum 16. Jahrhundert schon viel geringer sind. Zudem thematisiert und demonstriert er überzeugend die fehlende Systematik und Inkonsequenz einzelner sprachlicher und orthographischer Phänomene, was für diese Epoche kennzeichnend ist, wie etwa das Schwanken zwischen älteren und neueren phonetischen Formen („gleich“ vs. „geleich“, „rucken“ vs. „ruckhen“ u. „ruckchen“, „und“ vs. „undt“, „leib“ vs. „laib“). Das ,y“-Zeichen steht für ein langes ,i“ („dy“ - die, „sy“ - sie, „wy“ - wie), manchmal aber auch für kurzes („wyr“ - wir). Ein oft auftretendes Merkmal ist zudem die fehlende Endsilbe ,en“, vor allem bei Adjektivflexion („dy grosz waiss undt ploss fussen“). Die Schreibweise der großen und kleinen Anfangsbuchstaben steht ebenfalls noch nicht fest (etwa bei „Kertzen“ und „Thus“ im Gegensatz zu „knie“, „tuechel“ oder „maur“). Typisch ist auch die sogenannte Konsonantenhäufung, ${ }^{5}$ etwa bei „erstaunntt“ oder „auff“. 6

Auch wenn Doderers Text zumeist sehr authentisch erscheint, so finden sich in manchen Fällen Anpassungen zugunsten eines besseren Leseverständnisses. Manchmal verwendet er obendrein sprachliche Mittel, die in der geschilderten Epoche schon veraltet oder zu der Zeit noch ungebräuchlich waren.

Die Anpassungen, die den Text archaisieren, die also sprachlich in eine frühere sprachhistorische Epoche passen würden, dienen sichtlich der Figurencharakterisierung. Der Verfasser des Texts, Ruodlieb, erweist sich durch sie nämlich als gebildeter und belesener Mensch, der zudem oft dazu tendiert, archaische Textmittel und veraltete Strukturen, die als Lektürefrüchte kenntlich sind, zu benutzen. ${ }^{7}$ (Zur Zeit der Entstehung des Manuskripts ist er übrigens schon 69 Jahre alt.) ${ }^{8}$

Hierzu gehört beispielsweise die strikte Verwendung des Präteritums, obwohl um die Wende des 15. zum 16. Jahrhundert schon mindestens die Hälfte der Vergangenheitsformen periphrastisch (mit Perfekt) gebildet wurde. ${ }^{9}$ Auch die Schreibweise „smecken“ (statt schmecken) und „slueg“ (statt schlug) war im Frühneuhochdeutschen schon veraltet, ebenso die Verwendung des Buchstabens , $c^{6}$ im Auslaut, wo heute ein ,g'steht wie bei „lanc“ (lang) oder „lac“ (lag). Genauso verhält es sich auch bei Auslautverhärtungen, die, obwohl bis heute in der gesprochenen Sprache natürlich realisiert, im

$5 \mathrm{Zu}$ dieser Verwendung von Doppelkonsonanten ohne eigentlichen Lautwert vgl. Hartweg, Frédéric G.: Frühneuhochdeutsch: Eine Einführung in die deutsche Sprache des Spätmittelalters und der frühen Neuzeit. Tübingen 1989, S. 69.

6 Sämtliche Zitate in diesem Textabsatz finden sich in Doderer, Die Dämonen (Anm. 1), S. 779.

7 Informationen (unter anderem) dazu finden sich in den in eckigen Klammern eingeschlossenen Anmerkungen des Historikers René von Stangeler.

8 Vgl. Doderer, Die Dämonen (Anm. 1), S. 730.

9 Vgl. Hartweg, Frühneuhochdeutsch (Anm. 5), S. 177. 
Frühneuhochdeutschen immer seltener schriftlich notiert wurden, im Text aber recht oft thematisiert werden, etwa bei „undt“, „klait“ oder „handt“. ${ }^{10}$

Schließlich zeigt sich, dass die Textgestalt auch durch seine territoriale Herkunft beeinflusst ist. Bereits zu Beginn des Kapitels wird mitgeteilt, dass die Geschichte in Augsburg niedergeschrieben wurde. Der Sprachgebrauch zeigt jedenfalls Entsprechungen zu dem zu der Zeit dort gebräuchlichen Dialekt - so erscheint im Text etwa wiederholt ein ,p“ im Anlaut („prust“, „pindt“, „pald“), was für die südlichen Gebiete des deutschen Sprachraums typisch ist. ${ }^{11}$

Beim Übersetzen des Texts ergaben sich zunächst geringfügige Probleme beim primären Verständnis, die vor allem einzelne lexikalische Besonderheiten betreffen. Auch die phonologischen Unterschiede und die im Frühneuhochdeutschen üblichen Schreibungen führen zu Schwierigkeiten beim Lesen; nach ein paar Seiten tritt jedoch eine Gewöhnung ein und für Muttersprachler dürfte die Lektüre dann weitgehend problemlos sein. Es bleibt allerdings die Frage, wie der Text möglichst originalgetreu ins Tschechische übertragen werden könnte.

Die Handschrift stammt angeblich aus dem Jahr 1517, aus der Sprachepoche des Frühneuhochdeutschen. In der tschechischen Literatur gab es parallel dazu mit dem tschechischen Humanismus eine Blütezeit der tschechisch geschriebenen Literatur, so dass zahlreiche Quellen erhalten sind, aus denen die typischen Merkmale der damaligen Sprache übernommen werden können. Es konnte also ein tschechisches Werk aus dieser Epoche als Muster verwendet werden, um sich bei der Übersetzung auf die darin vorgefundene lautliche Gestalt, den syntaktischen Aufbau und dessen lexikalische Merkmale zu stützen. Bei der Auswahl eines geeigneten Werks spielte einerseits die Textgattung eine Rolle: Es sollte eine Erzählprosa sein, kein kanzleisprachlicher oder philosophischer Text, andererseits sollte das Werk auch für heutige Leser leicht verständlich sein.

Einen um das Jahr 1517 herum entstandenen Text als Gestaltungsvorbild zu verwenden, etwa einen von Řehoř Hrubý z Jelení oder Viktorin Kornel ze Všehrd, erwies sich jedoch nicht als die beste Wahl. Es zeigte sich, dass eine Übersetzung auf einer solchen Grundlage auf Leser wesentlich altertümlicher wirken würde als der Ausgangstext in Doderers Roman, weil die Entfernung zwischen dem von Doderer verwendeten und dem heutigen Deutschen geringer ist, als jene zwischen dem heutigen Tschechischen und dem des Jahres 1517. Deswegen diente ein später entstandenes Werk, die Kronika česká von Václav Hájek z Libočan als Gestaltungsvorbild. Diese Chronik erschien 1541, ist also 24 Jahre jünger als Doderers fingiertes Manuskript, und wurde von Hájek in einem volkstümlichen Stil abgefasst; der Eindruck der Altertümlichkeit beim Lesen entspricht recht gut der Wahrnehmung bei der Lektüre der Handschrift aus den Dämonen.

Naturgemäß ergaben sich beim Übersetzen selbst weitere Probleme, etwa angesichts der in eckigen Klammern in der Handschrift erscheinenden Anmerkungen, welche die Über-

10 Vgl. Hartweg, Frühneuhochdeutsch (Anm. 5), S. 145-147.

11 Vgl. Wiesinger, Peter: Schreibung und Aussprache im älteren Frühneuhochdeutschen. Zum Verhältnis vom Graphem - Phonem - Phon am bairisch-österreichischen Beispiel von Andreas Kurzmann um 1400. Berlin u. New York 2011, S. 192. 
setzungshilfen und sonstigen Hinweise (etwa Erklärungen zu außertextlichen historischen Realien) reproduzieren, die der junge Historiker René von Stangeler seinem Auftraggeber Jan Herzka gibt, während er ihm das Manuskript Ruodliebs vorliest. Diese Parenthesen stets an gleicher Stelle in der tschechischen Übersetzung zu setzen, war nicht durchgehend möglich. Wo beispielsweise im deutschen Original auf das frühneuhochdeutsche Wort „ân“ die Erklärung „[,ohne']“ folgt, wäre eine entsprechende Übersetzungshilfe in der tschechischen Übertragung aufgrund der klaren Verständlichkeit des Wortes unpassend gewesen. Um einen Ausgleich für Entfallenes zu schaffen, wurde versucht, Charakter, Zahl und Umfang der Anmerkungen zu erhalten, indem zusätzliche Anmerkungen nach Worten oder Ausdrücken ergänzt wurden, die in den Dämonen unkommentiert geblieben waren.

Der Ausschnitt, der zur Übersetzung ausgewählt wurde, eine zentrale Szene in Ruodliebs Manuskript, beschreibt die erste ,Folterung" der Bürgermeisterwitwe. Der Anblick ihres entkleideten Körpers ist für Ruodlieb mit starken Emotionen verbunden, die, wie er berichtet, bei ihm zu Verwirrungszuständen („nahent von sinnen“ - „nam mir mein atem und verstandt“ - „all erfuellt von der fraun“), Gang- und Standunsicherheit („,vermocht mich auff mein fussen niecht gehalten“ - „mir waren dy knie als zittern undt flatern under mir“) und vasomotorischer Instabilität („mir wardt noch mer heisz“) führen. Seine beängstigenden Wahrnehmungen des eigenen Körpers, die eine drohende Ohnmacht anzeigen, und seine intensiven Geruchs- und Gesichtsempfindungen (,irn weiszen leib“, „gesmach von den Kertzen undt dem Thus“, „vermaint ich gar, ich koennat sy auch smecken“) wirken zwar überzogen, werden aber verständlich, wenn man berücksichtigt, dass er laut eigener Aussage noch nie zuvor eine nackte Frau gesehen hat. ${ }^{12}$

Ruodliebs visuelle Wahrnehmungen werden von der Präsenz der Farbe Weiß dominiert; allein auf Seite 779 der Dämonen erscheint sie sechsmal. Nach Henner Löfflers $D o$ derer- $A B C$ ist Weiß in den meisten Werken Doderers „eine - nicht notwendigerweise die einzige, aber die primäre - Chiffre von Erotik "; ${ }^{13}$ und auch in dieser Episode scheint dies zuzutreffen. Wenig überraschend dient das Farbattribut weiß jedoch vor allem der Beschreibung des nackten Körpers der Bürgermeisterin: „dy grosz waiss undt ploss fussen von der fraun“, „auf irn weiszen leib“, „waiss ueber waiss von ir erm undt schuldern“, „der fraun an der seul irn weiszen leib“; einmal wird auch ihr „underklait“ so bezeichnet. Die Farbe von Gewand und Körper erscheint überdeutlich, die weiße Haut des Frauenkörpers wirkt beinahe blendend.

Bei alledem ist festzuhalten, dass eine dem Stadtpatriziat angehörende Frau wie die Bürgermeisterwitwe in Ruodliebs Manuskript typischerweise weder einer körperlichen Arbeit im Freien nachging, noch sich zum Vergnügen leicht bekleidet der Sonne aussetzte. Blasse Haut entsprach nicht nur dem weiblichen Schönheitsideal der Zeit, sie galt (nicht nur im 16. Jahrhundert) auch als Statussymbol und Ausweis persönlicher Vornehmheit - ebenso wie weiße (das heißt: aufwendig rasengebleichte und also teure) Leibwäsche. Ruodliebs wiederholte Betonung der weißen Haut der Agnes Stoecherin

12 Vgl. Doderer, Die Dämonen (Anm. 1), S. 768.

13 Löffler, Henner: Doderer-ABC. Ein Lexikon für Heimitisten. München 2000, S. 157. 
ist also in erster Linie sicherlich dem starken erotischen Reiz einer nackten und für ihn sonst unerreichbaren Frau geschuldet; zugleich zeugt sie aber auch von der kulturgeschichtlichen Sachkenntnis des Autors und promovierten Historikers Doderer, der - in einem offen sexuellen Kontext - bei seiner Gestaltung gewiss keiner „Chiffre von Erotik“ bedurfte, sondern mit der weißen Haut so simpel wie zutreffend ein zentrales Merkmal des aktuellen weiblichen Schönheitsideals fokussierte.

Zum Abschluss dieses Beitrags seien hier noch der kurze Ausschnitt aus Ruodliebs Manuskript und der Versuch seiner Übersetzung ins Tschechische vergleichend präsentiert:

Er wendt sich zue der truchen, soviel sach ich, wann ich geleich nahent von sinnen war, undt geb mir in die handt, ich soll dy fraun slahen, damit auf irn weiszen leib, undt ich konnt erkenn, daz es alles sammet [,Samt“] war, und ich slueg zue ettwas auff irn ruckchen undt suenst, aber sy duldtes [,duldete es“] ân [„ohne“] klag undt seufzat nur ir schemlichkeit wegen; wann man kunt mit den sametten Zeug kain smercz ir zuefuegen, hett man geleich harte zuegeslagen. Undt der Heimo, der slueg sy auch ein wenig, aber er spott sy mer noch in mannicher weis, auch mit woertten, dy ich niecht wil genennen. Undt ich vermocht mich auff mein fussen niecht gehalten und liesz ab und stellt mich an dy maur undt mir waren dy knie als zittern undt flatern under mir undt wurdten weich wy ain tuechel, so man in den windt gehengt hett, dannoch sach ich immer auff der fraun an der seul irn weiszen leib und mir wardt noch mer heisz undt der michel [„stark“] gesmach von den Kertzen undt dem Thus nam mir mein atem und verstandt [„Atem und Besinnung“] undt war all erfuellt von der fraun, do vermaint ich gar, ich koennat sy auch smecken. Der Heimo het sy darnach losgemachet undt ruefft mir wyder, doch mocht ich nicht gevolgen und nahent bei gehen. So fuert er sy ab von der seul und hinden an maur mit dem ruckhen; undt ich erstaunntt ueber dy purgermaistrin wy sy jetzt voller demuetiglichkait sich ertzeiget undt wehrat sich niecht [...]; darnach ziecht er ir die erm hoch auff ein hackchen ueber ir in der maur. Und gie umb sy her und
Obrátil se k truhle, toliko jsem viděl, byv právě takměř nesmyslný [,,beze smyslü“], a dal mně do ruky, že tím tu ženu mám bíti, na její bílé tělo, a já si všimnouti mohl, že to všeckno byl aksamit [„samet“], a trochu jsem ji bil na záda a jinde, ale ona to bez naříkání strpěla, jen kvůli hanbě své vzdychajíc; neb člověk jí nemohl tou aksamitovou věcí nižádnou bolest přivoditi, i kdyby velmi tvrdě byl uhodil. A Heimo, ten ji taktéž trochu bil, avšak ještě více on se jí ruozným zpuosobem posmieval, i slovy, jež nechci jmenovati. A já nemoha se na svých nohou udržeti, i pustil jsem a ke stěně se postavil a kolena se mi pode mnú klepala a třásla a změknula jako šátek, jež by byl do větru pověsil, alevšak na ženě u slúpu její bílé tělo porád jsem viděl a bylo mi ještě víc horko a mocná chutnost [,vůně“] od sviecí a kadidla vzala mi muoj dech i rozum, i byl jsem celek plný tej ženy, až jsem téměř sobě myslel, že ji i chutnati mohu. Heimo rozvázav ji znova mě zavolal, čehož však jsem nemohl následovati a bliže přijíti. Tu odvedl ji od slúpu a dozadu zády ke zdi; a já užasnuv nad purkmistrovú, kterak ona ted' plna poníženosti [„pokory“] se ukázala a se nebránila [...]; on pak ruce jí na hák nahoru nad ní vytáhl. A kol ní obcházeje, ji pozoroval, a náhle i já jsem blíže přišel a nemoha z ní pohled spustiti, nesmírně krásná se mi býti zdála, měla oči zavřené, tváří svou se obrátivši [„odvrátivši“]. 
beschaut sy do, undt gaechling kom ich auch nahent undt konnt mit main plick nicht mer von ir lassen, schien mir ân [„ohne“] maszen schoene, sy het ir augen geszlozzen und mit irn antlitz sich abgewendt. $^{14}$

\section{Quellen- und Literaturverzeichnis}

BAUFELD, Christa (1996): Kleines frühneuhochdeutsches Wörterbuch: Lexik aus Dichtung und Fachliteratur des Frühneuhochdeutschen. Tübingen.

DODERER, Heimito von (1995): Die Dämonen. Nach der Chronik des Sektionsrates Geyrenhoff. München.

HÁJEK Z LIBOČAN, Václav (2013): Hrsg. v. Jan Linka. Kronika česká. Praha.

HARTWEG, Frédéric G. (1989): Frühneuhochdeutsch: Eine Einführung in die deutsche Sprache des Spätmittelalters und der frühen Neuzeit. Tübingen.

LÖFFLER, Henner (2000): Doderer-ABC. Ein Lexikon für Heimitisten. München.

WIESINGER, Peter (2011): Schreibung und Aussprache im älteren Frühneuhochdeutschen. Zum Verhältnis vom Graphem - Phonem - Phon am bairisch-österreichischen Beispiel von Andreas Kurzmann um 1400. Berlin u. New York.

Internetquellen

Vokabulář webový, http://vokabular.ujc.cas.cz (31. 1. 2019)

Mgr. Anna Kovaříková / 407021@mail.muni.cz

Masarykova univerzita, Filozofická fakulta, Ústav germanistiky, nordistiky a nederlandistiky

Arna Nováka 1, 60200 Brno, CZ

14 Vgl. Doderer, Die Dämonen (Anm. 1), S. 779 f. 
